\title{
Multiscale processes of the flow and non- equilibrium interphase heat and mass transfer self-organization in multiphase systems
}

\author{
Vladimir $V$. Kuznetsov ${ }^{* 1,2}$ \\ ${ }^{1}$ Kutateladze Institute of Thermophysics SB RAS, 630090 Novosibirsk, Russia \\ ${ }^{2}$ Novosibirsk State University, Novosibirsk, 630090 Russia
}

\begin{abstract}
Multiscale processes of self-organization of the flow and nonequilibrium heat and mass transfer during the separation of a binary mixture in a multiphase system formed by a structural packing and a counter-flow vapor-liquid flow are considered. The conditions for the appearance of large-scale convective flow during separation of the binary mixture of refrigerants R21-R114 in a distillation column with Koch $1 Y$ structure packing are obtained. The physical properties of this mixture are similar to the properties of air component at cryogenic temperatures.
\end{abstract}

\section{Introduction}

One of the main problems hindering the development of high-efficiency multiphase heat and mass transfer equipment with unique characteristics on the specific surface and the intensity of heat and mass transfer processes is the multiscale flow, caused by the interaction of processes at the micro- and macrolevel. For such equipment, the selforganization of the gas-liquid flow determines the intensity of heat and mass transfer processes, both at the microscale and the macro scale, due to blocking of the heat and mass transfer surface during the development of large-scale flow unevenness.

An example of multiphase heat and mass transfer equipment, when a high level of the specific surface and the intensity of heat and mass transfer processes are essential for increasing the productivity, are the distillation columns and two-phase compact heat exchangers [1]. Distillation is the most effective way for multicomponent mixture separation into pure components differing in boiling points, and it is carried out during counter-current contact of vapor and liquid [2]. Recently, a problem of increasing of the productivity of distillation columns has necessitated the use of structural packing to create a direct contact between the vapor and liquid. These packings provide less pressure loss and increase the phase contact surface compared to irregular packing or trays. For structure packing application, the physical processes in complex channel systems are studied, as well as methods for calculating the efficiency of binary mixture separation for the packing of various types, developed in [3].

* Corresponding author: vladkuz@itp.nsc.ru 
The process of vapor enrichment with volatile distillate fraction and their depletion of liquid with the release of separation products in the upper and lower parts of the column is nonequilibrium. It occurs due to the difference in a concentration of the components in the liquid and vapor. Therefore, the occurrence of large-scale unevenness in the flow and fraction concentration for the liquid and gas causes the deterioration in the separation efficiency [4, 5]. In this paper, the processes of self-organization of the flow and nonequilibrium heat and mass transfer during separation of the binary mixture in complex channels systems of the structure packing and their influence on the process of separation of the R21-R114 binary mixture in a column filled with the Koch 1Y structure packing are considered. The physical properties of this mixture are similar to the properties of air components at cryogenic temperature.

\section{Multiscale processes of counter-current gas-liquid flow self- organization in structure packing}

Let us consider the counter-current flow of liquid and vapor in the distillation column filled with structure packing during binary gas mixture separation. In such a column, the liquid flows down under the action of gravity in the form of a thin film over the surface of structure packing, and the vapor rises upward under the conditions of nonequilibrium heat and mass transfer at the interphase. Structure packing represents the corrugated perforated sheets, tightly pressed together. It forms a complex system of channels for the vapor flow. The presence of a microstructure on the surface of the packing increases the wettability and uniformity of the flow as far as the heat and mass transfer coefficient for the liquid phase. The use of structure packing allows obtaining the specific surface area up to $750 \mathrm{~m}^{2} / \mathrm{m}^{3}$ with porosity of 0.98 , and intense heat and mass transfer at a micro level that is achieved by a small thickness of the liquid film and spontaneous twist of the vapor flow.

The system under consideration is multiphase with fixed solid phase and countercurrent vapor-liquid flow in the packing channels. The main cause of the spontaneous selforganization of the counter-current multicomponent flow with the formation of large-scale structures in complex channel system of the structure packing during the separation process is the convective flows arising in the column with a negative vapor density gradient along the gravity vector. The reduction of gas density is conditioned by the transition of the volatile distillate fraction from the liquid to vapor due to the mass transfer process.

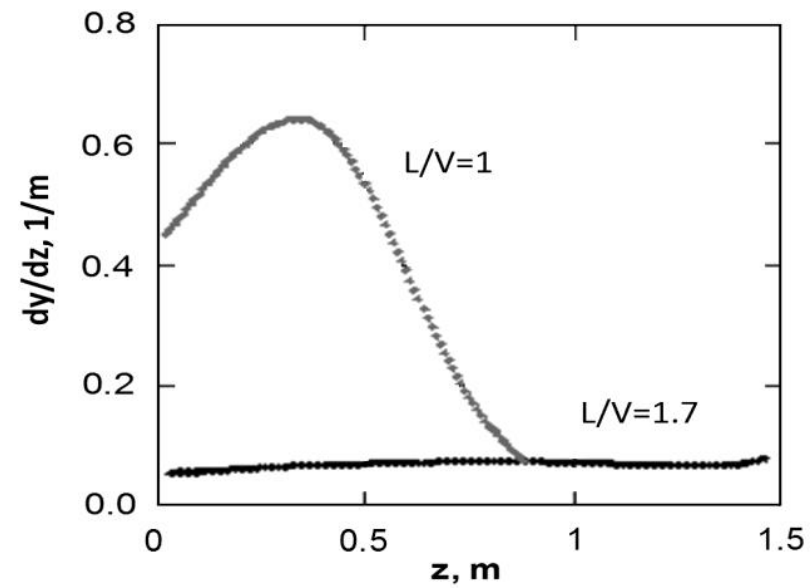

Fig. 1. Variation of the concentration gradient of volatile distillate fraction along the gravity vector during separation of R21-R114. 
Numerical calculations based on the one-dimensional non-equilibrium separation model [6], used for the separation of R21-R114 mixture in a column filled with Koch 1Y structure packing for the conditions corresponding to experiments [4], showed that the highest concentration gradient of the volatile distillate fraction and vapor density are located in the top of the column. The concentration gradient, shown in Fig. 1, increases significantly with an increase in the ratio of molar flow rates of liquid and vapor from 1 to 1.7 that may be the cause of the convective flow development in the gravity field. Here the coordinate $z$ is measured from the top of the column.

Let us consider the effect of negative gas density gradient on the stability of the flow in a column. During binary mixture separation, the gas density is changed along the column due to heat and mass transfer between liquid and gas. It is typical for separation that the gas mixture density depends mainly on the concentration of volatile distillate fraction because the temperature is varied slightly. Assuming small variation in the density of gas mixture along the column, we obtain:

$$
\rho_{G}=\rho_{G 0}+\rho_{G}^{\prime}, \quad \rho_{G}=\rho_{G}\left(Y_{G}, T\right), \rho_{G}=\rho_{G 0}+\left(\partial \rho_{G} / \partial Y_{G}\right)_{T} Y_{G}^{\prime},
$$

where $Y_{G}=Y_{G 0}+Y_{G}^{\prime}$ is the concentration of volatile distillate fraction. Introducing parameter $\beta_{\mathrm{Y}}=-\left(\partial \rho_{G} / \partial Y_{G}\right)_{T} \rho_{G 0}^{-1}$, we obtain the averaged momentum equation for the gas as follows:

$$
\left\langle\left(\mathbf{u}_{\mathrm{G}} \nabla\right) \mathbf{u}_{\mathrm{G}}\right\rangle=-\frac{\nabla \mathrm{p}_{\mathrm{G}}}{\rho_{\mathrm{G} 0}}+\left\langle v_{\mathrm{G}} \Delta \mathbf{u}_{\mathrm{G}}\right\rangle-\beta_{\mathrm{Y}} Y_{\mathrm{G}}^{\prime} \mathbf{g},
$$

where $\mathbf{u}_{\mathbf{G}}$ is the velocity vector. Scaling equation (2) by the characteristic size $l$, the characteristic gas velocity $U_{\mathrm{G}}$, the characteristic concentration $Y_{0}=l \cdot d Y / d z$ we obtain:

$$
\left\langle\left(\mathbf{u}_{\mathrm{G}} \nabla\right) \mathbf{u}_{\mathrm{G}}\right\rangle=-\nabla \widetilde{\mathrm{p}}_{\mathrm{G}}-N_{\text {conv }} \widetilde{Y}_{\mathrm{G}} \frac{\mathbf{g}}{g}+\frac{1}{\operatorname{Re}}\left\langle\nabla^{2} \mathbf{u}_{\mathrm{G}}\right\rangle
$$

Here the dimensionless number that determines the ratio of gravitational and inertial forces in a convective flow and called as convection number is introduced as follows:

$$
N_{c o n v}=\frac{l^{2} g \beta_{Y} d Y / d z}{U_{G}^{2}}=\frac{R a \cdot \operatorname{Pr}}{P e^{2}}
$$

The convection number $N_{\text {conv }}$ determines the ratio of gravitational and inertial forces during ascending vapor flow. Therefore, unstable vapor flow regimes correspond to the convection number, which is much higher than unity. The calculations of the separation of binary refrigerants mixture show that the convection number $N_{\text {conv }}$ can significantly vary along column length. Figure 2 shows the variation in the convection number $N_{\text {conv }}$ along the gravity vector for the molar liquid to vapor ratios $L / V=1$ and $L / V=1.7$. The perturbations with characteristic size $l$ equal to the diameter of column D are considered in calculations.

As can be seen, the convection number considerably exceeds unity for the columns with a diameter exceeding 0.2 meters if $L / V=1$. For this case, one can expect the appearance of convective flows in the column and significant heterogeneity of the components concentration in the column cross-section, which was observed experimentally in $[4,5]$. 


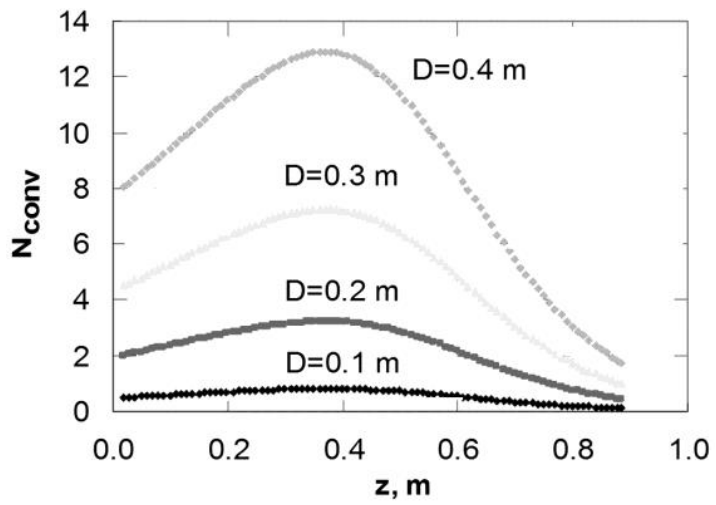

a)

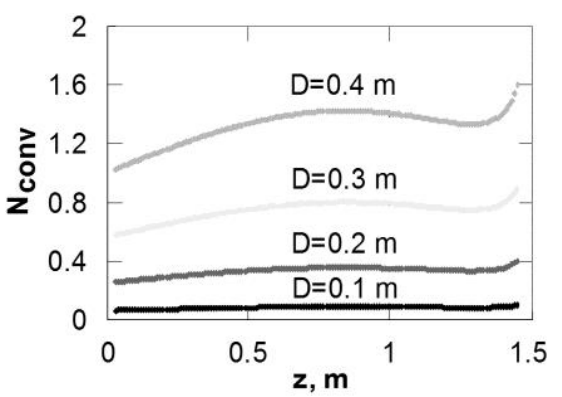

b)

Fig. 2. Variation of the convection number along the column: a) $L / V=1$; b) $L / V=1.7$.

\section{Conclusions}

The obtained results allow characterizing the conditions for the development of large-scale convective structures during counter-current vapor-liquid flow in structure packing, as an example of the multiphase system with a fixed solid phase. It is necessary for the development of highly efficient heat and mass transfer devices. It is established that the ratio of the liquid to vapor molar flows in a distillation column with a structural packing closed to unity is the most dangerous and can lead to the development of convective flow in the large-diameter column with a negative gradient of the vapor density along the direction of the gravity vector. In this case, the separation efficiency will decrease because of the occurrence of large-scale heterogeneity in the concentration of mixture components.

This work was performed in Kutateladze Institute of Thermophysics SB RAS by a grant from the Russian Science Foundation (project No. 14-49-00010).

\section{References}

1. V.V. Kuznetsov, A.S. Shamirzaev, J. Eng. Thermophys. 24, 357 (2015)

2. M.P. Malkov, I.B. Danilov, A.G. Zel'dovich, A.B. Fradkov, Handbook of Physicotechnical Principles of Cryogenics (Energoatomizdat, Moscow, 1985)

3. B. Hanley, C.C. Chen, AIChE J. 58, 132 (2012)

4. A.N. Pavlenko, N.I. Pecherkin, V.Yu. Chekhovich, V.E. Zhukov, S. Sunder, P. Houghton, A.F. Serov, A.D. Nazarov, Theor. Found Chem. Eng. 40, 329 (2006)

5. A.N. Pavlenko, X. Li, V.E. Zhukov, N.I. Pecherkin, O.A. Volodin, A.S. Surtaev, X. Gao, L. Zhang, H. Sui, H. Li, J. Eng. Thermophys. 24, 210 (2015)

6. R. Taylor, R. Krishna, Multicomponent Mass Transfer (Wiley - IEEE, 1993) 